\title{
MODÉLISATION DES CARACTÉRISTIQUES DU GaAs MESFET
}

\author{
C. KenZAI ${ }^{a}$, M. ZAABAT ${ }^{a}, \mathrm{Y} \mathrm{SAIDI}^{a}$ AND A. KHITER ${ }^{b}$ \\ ${ }^{a}$ Institut de Physique, Université de Constantine, 25 000, Algérie \\ ${ }^{b}$ Institut des Sciences Exactes, Université de Batna, 05 000, Algérie \\ (Received June 15, 1999; revised version April 11, 2000)
}

A NEW MODELING OF THE CHARACTERISTICS OF GaAs MESFET'S: A new approach for $I-V$ standard model is proposed. This approach allowed to conceive applicable model for MESFET's operating in the turn-one or pinch-off region, and valid for the short-channel and the long-channel MESFET's, in which the two-dimensional potential distribution contributed by the depletion layer under the gate is obtained by conventional ID approximation. The drain current is decomposed into two components; the first is due to the conduction current flowing through the conduction channel, and the second component is a result of the current flowing through the space charge region resulting from the injection of the channel electrons into this last region. Moreover, comparison between the proposed analytical model and the experimental data are made and good agreement is obtained. In the end, this model is applied to estimate the cut-off frequency of the MESFET-GaAs from these static characteristics. This estimation is based on the theoretical calculation of the transconductance and the gate capacitance of the device.

PACS numbers: $85.30 .-\mathrm{z}$

\section{Introduction}

Le transistor à effet de champ à grille de Schottky à l'arséniure de gallium, connu par son abréviation anglaise MESFET (metal semiconductor field effect transistor) a été utilisé dans les deux dernières décennies dans les applications microondes; du fait de sa facilité de fabrication, sa rapidité de réponse et sa performance faible bruit.

Tout d'abord nous présentons un modèle simplifié où la zone de charge d'espace (ZCE) est parfaitement dépeuplée. Ensuite l'originalité de notre étude est de proposer une nouvelle approche pour le calcul du courant de drain, qui tient compte de l'effet de l'injection de porteurs majoritaires dans la zone de charge d'espace. 
Dans cet article on va proposer un modèle analytique qui décrit le fonctionnement électrique statique du MESFET, quelque soit sa longueur du canal $(L)$ et quelque soit son régime de fonctionnement (pincement ou non). Ce modèle tient compte de la variation de l'intensité du champs électrique le long du canal, et de l'effet de l'injection des électrons de conduction du canal dans la ZCE, ce qui nous. permet de calculer la valeur du courant de drain en régime de pincement. L'effet des résistances parasites de source et de drain $\left(R_{\mathrm{S}}\right.$ et $\left.R_{\mathrm{D}}\right)$ est pris en considération dans le développement de ce modèle.

Dans Sec. 2, nous déterminons le potentiel et le champ électrique à deux dimensions (2D) dans la ZCE dûs à la charge électrique formée sous la grille qui peuvent être obtenus en résolvant l'équation de Poisson par l'approximation conventionnelle 1D. Dans Sec. 3, on calcule le courant de drain $I_{\mathrm{D}}$, comprenant deux parties: la première $I_{\mathrm{DC}}$ due au courant qui passe à travers le canal conducteur, et la deuxième $I_{\mathrm{DZCE}}$ due au courant qui passe à travers la ZCE. Le calcul de la fréquence de coupure à partir de ce modèle est présenté au Sec. 4. Dans Sec. 5, les caractéristiques $I-V$ obtenus par ce modèle en utilisant 3 expressions différentes de la vitesse des électrons $v(E)[1-3]$ pour des MESFET's-GaAs de différentes longueurs de canal $L$ sont présentés, ainsi que les résultats de simulation donnant la variation de la capacité de grille et la fréquence de coupure en fonction de la tension de grille $V_{\mathrm{G}}$. La conclusion est donnée dans Sec. 6 .

\section{Calcul du potentiel et du champ électrique}

La modélisation du MESFET exige souvent plusieurs hypothèses simplificatrices dues aux difficultés imposées par les effets de bord [4,5]. Pour des raisons de clarté on va restreindre la modélisation à la région active du canal. On considère alors la structure du MESFET à dopage $n$ représentée sur Fig. 1 .

Lorsque tous les donneurs sont supposés ionisés à la température ambiante, le potentiel électrique dû à la charge électrique formée sous la grille peut être donné par [6]:

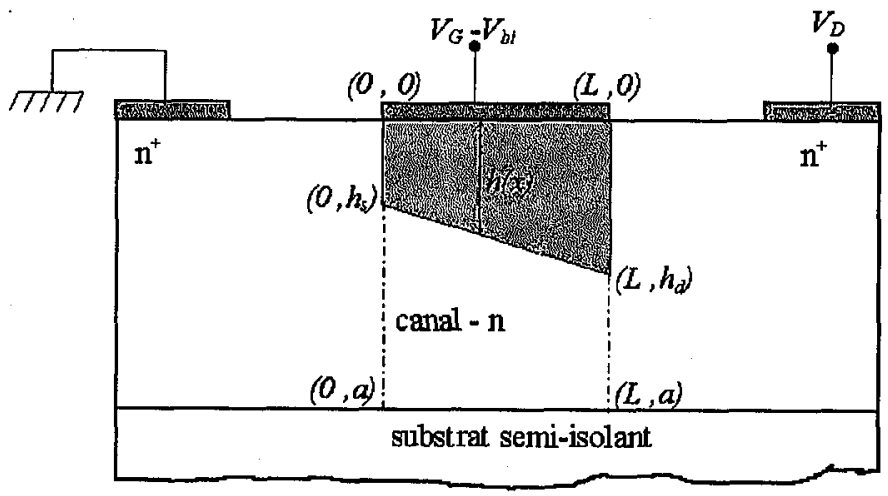

Fig. 1. Schéma du MESFET. 


$$
\Psi(x, y)=\frac{1}{\varepsilon} \int_{0}^{y} q N_{\mathrm{D}}\left(x, y^{\prime}\right) y^{\prime} \mathrm{d} y^{\prime}+\frac{1}{\varepsilon} y \int_{y}^{h(x)} q N_{\mathrm{D}}\left(x, y^{\prime}\right) \mathrm{d} y^{\prime}+V_{\mathrm{G}}-V_{\mathrm{bi}},
$$

où $\varepsilon_{0}$ - permittivité du vide, $\varepsilon_{\mathrm{GaAs}}$ - constante diélectrique GaAs, $V_{\mathrm{G}}$ - la tension de la grille, $y$ - la direction transversale et $h(x)$ - épaisseur de la zone de charge d'espace en un point $x$ du canal avec $N_{\mathrm{D}}(x, y)=N_{\mathrm{D}}(y)-n(x, y), N_{\mathrm{D}}(y)-$ la densité de donneurs qui peut varier avec $y$ et $n(x, y)$ - la densité des électrons libres dans la ZCE, $q$ - la charge de l'électron, $V_{\mathrm{bi}}$ - la tension du barrière Schottky et $\varepsilon=\varepsilon_{0} \varepsilon_{\mathrm{GaAs}}$. Notons que l'expression (1) est obtenue en se basant sur le fait que l'épaisseur de la ZCE, $h(x)$, varie lentement le long du canal. Le potentiel dans le canal conducteur $\Psi_{\mathrm{c}}(x, y)$ s'obtient en portant $y=h(x)$ dans (1), soit

$$
\Psi_{\mathrm{c}}(x, y)=\frac{1}{\varepsilon} \int_{0}^{h(z)} q N_{\mathrm{D}}\left(x, y^{\prime}\right) y^{\prime} \mathrm{d} y^{\prime}+V_{\mathrm{G}}-V_{\mathrm{bi}} .
$$

Pour un dopage homogène, et pour une ZCE vide de porteurs, on a $N_{\mathrm{D}}(x, y)=N_{\mathrm{D}}$, et Eq. (1) devient

$$
\Psi(x, y)=\frac{-q N_{\mathrm{D}} y^{2}}{2 \varepsilon}+\frac{q N_{\mathrm{D}} y}{\varepsilon} h(x)+V_{\mathrm{G}}-V_{\mathrm{bi}}
$$

et par consèquent

$$
\Psi_{\mathrm{c}}(x)=\frac{q N_{\mathrm{D}} h^{2}(x)}{2 \varepsilon}+V_{\mathrm{G}}-V_{\mathrm{bi}}
$$

Le champ électrique dans la ZCE se déduit par $E(x, y)=-\nabla \Psi(x, y)$ et soit

$$
\boldsymbol{E}(x, y)=-\frac{q N_{\mathrm{D}} y}{\varepsilon} \frac{\mathrm{d} h(x)}{\mathrm{d} x} i-\frac{q N_{\mathrm{D}}}{\varepsilon}[h(x)-y] \boldsymbol{j}
$$

et dans le canal conducteur on a

$$
E=E_{x}=-\frac{q N_{\mathrm{D}} h(x)}{\varepsilon} \frac{\mathrm{d} h(x)}{\mathrm{d} x} .
$$

La détermination du potentiel et du champ électrique exige la connaissance de $h(x)$. Dans la suite on se placera dans l'approximation conventionnelle du canal graduel, qui nous permet de déduire l'expression de $h(x)$ en fonction de $h_{\mathrm{s}}$ et $h_{\mathrm{d}}$; ses valeurs côté source et côté drain

$$
h(x)=\frac{h_{\mathrm{d}}-h_{\mathrm{s}}}{L} x+h_{\mathrm{s}} .
$$

Les calculs effectués ci-dessus, ignorent bien entendu la contribution de la ZCE se situant sous la surface libre dans le potentiel [2].

\section{Calcul du courant de drain}

L'expression (5) montre que le champ électrique dans la ZCE possède deux composantes. La composante longitudinale atteint sa valeur maximale en $y=h(x)$; ainsi les électrons de conduction du canal qui atteignent la limite de la ZCE vont alors trouver un champ électrique favorable qui les injecte dans cette dernière, là où ils rencontrent également un champ électrique favorable (Eq. (5)) qui les propulse vers l'électrode de drain en contribuant ainsi dans le courant de drain. De ce fait le courant de drain peut être exprimé en un point $x$ du canal par 


$$
I_{\mathrm{D}}(x)=q Z N_{\mathrm{D}} v_{x}[E(x)][a-h(x)]+q Z \int_{h_{\mathrm{s}}}^{h(x)} J_{x}(x, y) \mathrm{d} y,
$$

$Z$ est la largeur du canal. Le premier terme de (7) représente la partie du courant de drain passant à travers le canal conducteur, et le second représente la deuxième partie qui passe à travers la ZCE, avec une intégrale de $h_{\mathrm{s}}$ à $h(x)$, ce qui signifie qu'on a supposé que $J_{x}(x, y)=0$ pour $y<h_{\mathrm{s}}$. Le calcul de cette deuxième partie exige la connaissance de la densité $n(x, y)$ des électrons libres dans la ZCE. La determination de $n(x, y)$ peut étre obtenue par la résolution de l'équation de continuité qui prend la forme $(8)$ ci-dessous en régime stationnaire $(\partial n / \partial t=0)$

$$
\begin{aligned}
\frac{q}{K T} & {\left[\frac{\partial n(x, y)}{\partial x} E_{x}+\frac{\partial n(x, y)}{\partial y} E_{y}+n(x, y)\left(\frac{\partial E_{x}}{\partial x}+\frac{\partial E_{y}}{\partial y}\right)\right] } \\
& +\frac{\partial^{2} n(x, y)}{\partial x^{2}}+\frac{\partial^{2} n(x, y)}{\partial y^{2}}=0,
\end{aligned}
$$

$K$ est la constante de Boltzmann, et $T$ - la température absolue. Compte tenu de l'expression (3) une solution d'Eq. (8) peut être donnée [1] par

$$
n(x, y)=N_{\mathrm{D}} \exp \left\{\frac{q}{K T}\left[\frac{-q N_{\mathrm{D}} y^{2}}{2 \varepsilon}+\frac{q N_{\mathrm{D}} y}{\varepsilon} h(x)+V_{\mathrm{G}}-V_{\mathrm{bi}}\right]\right\} .
$$

Cette procédure s'avère exiger un calcul important du fait que $J_{x}$ comprend deux termes celui dû au champ électrique et celui dû au gradient de porteurs dans la ZCE. Pour cela nous avons développé une deuxième méthode qui consiste à supposer que le courant de drain est conservatif (tous les électrons qui sont injectés dans la ZCE, atteignent l'électrode de drain). Dans ce cas (Fig. 2), au lieu de calculer le courant qui traverse la section verticale $S^{\prime}=z\left[h(x)-h_{\mathrm{s}}\right]$, on calcule le courant qui traverse la section oblique $S=Z \sqrt{x^{2}-\left[h(x)-h_{\mathrm{s}}\right]^{2}}$, là où on connaît la densité des charges libres, à savoir $N_{\mathrm{D}}$. Ainsi le développement du courant de drain en $x=L$ permet d'obtenir [1]:

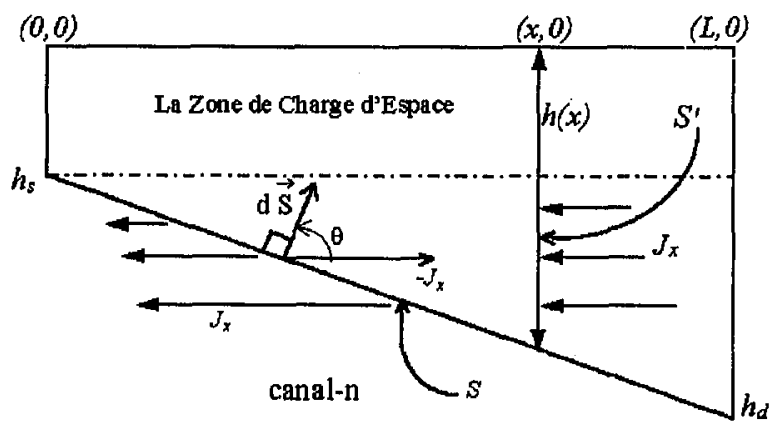

Fig. 2. Schéma illustratif représentant le courant traversant la ZCE. 


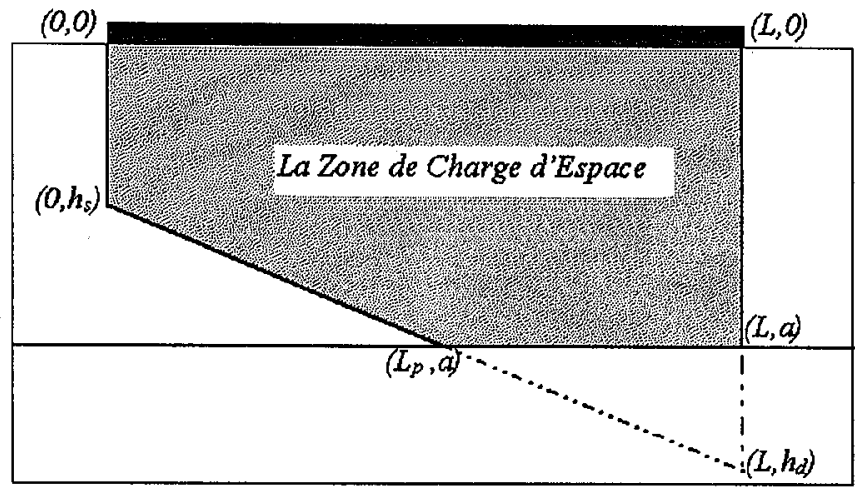

Fig. 3. Forme de la ZCE en régime de pincement.

$$
I_{\mathrm{D}}(x)=q Z N_{\mathrm{D}} v_{x}\left(L, h_{\mathrm{d}}\right)[a-h(x)]+q N_{\mathrm{D}} Z \frac{h_{\mathrm{d}}-h_{\mathrm{s}}}{L} \int_{h_{\mathrm{s}}}^{h(x)} v_{x}[x, h(x)] \mathrm{d} y .
$$

Cette expression (10) qui est valable lorsque $h_{\mathrm{d}}$ est inférieur à $a$, l'épaisseur de la zone active, cesse de l'être lorsque le composant fonctionne en régime de pincement $\left(h_{\mathrm{d}}>a\right)$. Afin que notre approche soit valable également en régime de pincement, on va falloir adapter l'expression (10). La première modification est évidente; elle consiste à mettre le premier terme égale à zéro. Pour le second terme l'intégrale de 0 à $L$ n'est plus valable car le point $\left(L_{p}, a\right)$ se déplace vers la source quand $V_{\mathrm{D}}$ augmente (Fig. 3). L'intégrale figurant devrait alors s'effectuer de 0 à $L_{p}$. Pour déterminer la valeur de $L_{p}$ on suppose comme auparavant que la ZCE possède une forme graduelle avec une profondeur "fictive" $h_{\mathrm{d}}>a$ du côté drain. Figure 3 nous permet de déduire l'expression de $L_{p}$ :

$$
L_{p}=\frac{a-h_{\mathrm{s}}}{h_{\mathrm{d}}-h_{\mathrm{s}}} L,
$$

d'où l'expression du courant de drain en régime de pincement

$$
I_{\mathrm{D}}=q N_{\mathrm{D}} Z \frac{h_{\mathrm{d}}-h_{\mathrm{s}}}{L} \int_{0}^{L_{\boldsymbol{p}}} v_{x}[x, h(x)] \mathrm{d} x .
$$

Le calcul de $I_{D}$ consiste alors à résoudre numériquement l'une des deux Eqs. (10) lorsque $h_{\mathrm{d}} \leq a$, et (12) dans le cas contraire. Sachant que

$$
\begin{aligned}
& h_{\mathrm{s}}=a \sqrt{\frac{V_{\mathrm{bi}}-V_{\mathrm{G}}+V_{0}}{V_{p}},} \\
& V_{0}=R_{\mathrm{S}} I_{\mathrm{D}}, \\
& h_{\mathrm{d}}=a \sqrt{\frac{V_{\mathrm{bi}}-V_{\mathrm{G}}+V_{1}}{V_{p}}}, \\
& V_{1}=V_{\mathrm{D}}-R_{\mathrm{D}} I_{\mathrm{DC}},
\end{aligned}
$$

$R_{\mathrm{S}}$ et $R_{\mathrm{D}}$ sont les résistances parasites de source et de drain respectivement. 
Notons que la variation de la vitesse des électrons en fonction du champ électrique dans le GaAs augmente avec $E$, et atteint une certaine valeur culminante pour une certaine valeur $E_{\mathrm{C}}$ du champ électrique, puis elle subit une décroissance pour $E>E_{\mathrm{C}}$. Cette caractéristique et le fait que l'intensité du champ électrique varie le long du canal, donnent lieu à une charge d'accumulation dans la région du canal dans laquelle $E>E_{\mathrm{C}}$ et une charge de déplétion dans la région dans laquelle $E<E_{\mathrm{C}}$. Cette propriété doit être prise en considération lorsqu'on utilise une expression de la vitesse donnant une telle allure, ce qui nous conduit à résoudre dans le canal, l'équation

$$
\mathrm{d} n(v)=0 \text {. }
$$

- La résolution de cette équation dans la partie du canal, dans laquelle $E>E_{\mathrm{C}}$ avec la condition $n\left(L_{1}\right)=N_{\mathrm{D}}$ et $v_{\mathrm{s}}=v\left(E=E_{\mathrm{C}}\right)=v\left(x=L_{1}\right)$, nous permet d'aboutir aux expressions du courant de drain suivantes:

$$
I_{\mathrm{D}}=q n\left(L_{1}\right) v_{\mathrm{s}} Z\left(a-h_{\mathrm{d}}\right)+q n\left(L_{1}\right) Z v_{\mathrm{s}}\left(h_{\mathrm{d}}-h_{\mathrm{s}}\right)
$$

en régime de conduction sans pincement,

$$
I_{\mathrm{D}}=q N_{\mathrm{D}} Z v_{\mathrm{s}}\left(h_{\mathrm{d}}-h_{\mathrm{s}}\right) \frac{L_{p}}{L}
$$

et en régime de pincement.

\section{Calcul de la fréquence de coupure}

La fréquence de coupure de MESFET est un paramètre caractéristique important. Elle est donnée [7] par

$$
f_{\mathrm{C}}=\frac{G_{m}}{2 \pi C_{\mathrm{G}}}
$$

avec $G_{m}$ - la transconductance, et $C_{\mathrm{G}}$ - la capacité de grille. La transconductance est donnée par

$$
G_{m}=\left.\frac{\partial I_{\mathrm{D}}}{\partial V_{\mathrm{G}}}\right|_{V_{\mathrm{D}}} .
$$

A partir des expressions du courant de drain établies dans la section précédente il est difficile de faire le calcul analytique de cette dérivée. En revanche, la méthode numérique permet de la calculer aisément

$$
G_{m}=\frac{I_{\mathrm{D}}^{i+1}-I_{\mathrm{D}}^{i}}{V_{\mathrm{G}}^{i+1}-V_{\mathrm{G}}^{i}}, \quad i \quad \text { indice entier. }
$$

La capacité de grille est donnée par

$$
C_{\mathrm{G}}=\left|\frac{\mathrm{d} Q}{\mathrm{~d} V_{\mathrm{G}}}\right|,
$$

$Q$ représente la charge électrique formée sous la grille. Elle peut être exprimée par

$$
Q_{1}=q N_{\mathrm{D}} Z \int_{0}^{L} h(x) \mathrm{d} x-q Z \iint_{S_{1}} n(x, y) \mathrm{d} x \mathrm{~d} y,
$$

quand $h_{\mathrm{d}}$ est inférieur à $a$, et

$$
Q_{2}=q N_{\mathrm{D}} Z \int_{0}^{L_{p}} h(x) \mathrm{d} x+q N_{\mathrm{D}} Z \int_{L_{p}}^{L} a \mathrm{~d} x-q Z \iint_{S_{2}} n(x, y) \mathrm{d} x \mathrm{~d} y,
$$

quand $h_{\mathrm{d}}$ "fictif" est supérieur à $a$. 
Le premier terme de (24) représente la charge de déplétion lorque la ZCE est vide de porteurs, et le second terme représente la charge due aux électrons libres qui y existent. C'est la même explication pour l'expression (25), qui est déduite de la forme de la ZCE représentée sur Fig. 3. Compte tenu de (6) et (9), le calcul des valeurs de ces deux expressions est possible. D'autre part, la méthode utilisée dans l'établissement de (10) nous permet d'obtenir [1]:

$$
n(x, y)=\frac{v_{x}[x, h(x)]}{v_{x}(x, y)} N_{\mathrm{D}}
$$

Dans ce cas le développement de (24) et (25) nous permet d'aboutir à

$$
\begin{aligned}
Q_{1}= & q N_{\mathrm{D}} Z L a \frac{d-s}{2}-q N_{\mathrm{D}} Z \int_{0}^{L}\left[\int_{h_{\mathrm{s}}}^{h(x)} f(x, y) \mathrm{d} y\right] \mathrm{d} x \\
Q_{2}= & q N_{\mathrm{D}} Z L a\left[1-\frac{1}{2} \frac{(1-s)^{2}}{d-s}\right]-q N_{\mathrm{D}} Z \int_{0}^{L_{p}}\left[\int_{h_{\mathrm{s}}}^{h(x)} f(x, y) \mathrm{d} y\right] \mathrm{d} x \\
& -q N_{\mathrm{D}} Z \int_{L_{p}}^{L}\left[\int_{h_{\mathrm{s}}}^{a} f(x, y) \mathrm{d} y\right] \mathrm{d} x
\end{aligned}
$$

avec $h_{\mathrm{s}}=a s, h_{\mathrm{d}}=a d$, et $f(x, y)=v_{x}[x, h(x)] / v_{x}(x, y)$. On pose

$$
C_{\mathrm{G} 1}=\left|\frac{\mathrm{d} Q_{1}}{\mathrm{~d} V_{\mathrm{G}}}\right|, \quad C_{\mathrm{G} 2}=\left|\frac{\mathrm{d} Q_{2}}{\mathrm{~d} V_{\mathrm{G}}}\right|,
$$

$C_{\mathrm{G} 1}$ et $C_{\mathrm{G} 2}$ sont alors déterminées respectivement à partir de (26) et (27):

$$
\begin{aligned}
C_{\mathrm{G} 1} & =\mid \frac{q N_{\mathrm{D}} Z L a}{4 V_{p} s d}\left[s+d+G_{m}\left(s R_{\mathrm{D}}-d R_{\mathrm{S}}\right)-G_{m}^{\prime} s R_{\mathrm{D}}\right] \\
& +q N_{\mathrm{D}} Z \frac{\mathrm{d}}{\mathrm{d} V_{\mathrm{G}}}\left\{\int_{0}^{L}\left[\int_{h_{\mathrm{s}}}^{h(x)} f(x, y) \mathrm{d} y\right] \mathrm{d} x\right\} \mid
\end{aligned}
$$

avec $G_{m}^{\prime}=\mathrm{d} I_{\mathrm{DZCE}} / \mathrm{d} V_{\mathrm{G}}$, d'où

$$
\begin{aligned}
& C_{\mathrm{G} 2}=\mid \frac{q N_{\mathrm{D}} Z L a}{4 V_{p} s d} \frac{1-s}{(d-s)^{2}} \\
& \times\left\{(2 d-s-1)\left(d-d R_{\mathrm{S}} G_{m}\right)+(1-s)\left[s+s R_{\mathrm{D}}\left(G_{m}-G_{m}\right)\right]\right\} \\
&+q N_{\mathrm{D}} Z \frac{\mathrm{d}}{\mathrm{d} V_{\mathrm{G}}}\left\{\int_{0}^{L_{\mathrm{p}}}\left[\int_{h_{\mathrm{s}}}^{h(x)} f(x, y) \mathrm{d} y\right] \mathrm{d} x+\int_{L_{p}}^{L}\left[\int_{h_{\mathrm{s}}}^{a} f(x, y) \mathrm{d} y\right] \mathrm{d} x\right\} \mid, \\
& C_{\mathrm{G}}= \begin{cases}C_{\mathrm{G} 1} & \text { si } h_{\mathrm{d}} \leq a, \\
C_{\mathrm{G} 2} & \text { si } h_{\mathrm{d}} \geq a .\end{cases}
\end{aligned}
$$

Le calcul de $G_{m}$ et de $C_{\mathrm{G}}$ nous permet par la suite de déduire la valeur de la fréquence de coupure par (20). 


\section{Résultats et discussions}

Pour examiner la validité des expressions du courant de drain établies dans Sec. 3 , on a besoin d'utiliser une expression convenable de la vitesse des électrons $v(E)$. On utilise tout d'abord l'expression suivante $[7,8]$ :

$$
v(E)=\frac{\mu_{\mathrm{n}} E}{1+\frac{\mu_{\mathrm{n}} E}{v_{\mathrm{s}}}}
$$

$\mu_{\mathrm{n}}$ est la mobilité des électrons à faible champ électrique, et $v_{\mathrm{s}}$ - leur vitesse de saturation. On utilise les données des transistors GAT1 $(L=4 \mu \mathrm{m})$ et THC $(L=1 \mu \mathrm{m})$ dont les paramètres sont donnés dans Tableau I.

TABLEAU I

Paramètres des transistors étudiés.

\begin{tabular}{c|c|c|c|c|c|c|c|c}
\hline \hline MESFET & $\begin{array}{c}L \\
{[\mu \mathrm{m}]}\end{array}$ & $\begin{array}{c}a \\
{[\mu \mathrm{m}]}\end{array}$ & $\begin{array}{c}Z \\
{[\mu \mathrm{m}]}\end{array}$ & $\begin{array}{c}N_{\mathrm{D}} \\
{\left[\mathrm{cm}^{-3}\right]}\end{array}$ & $\begin{array}{c}\mu_{\mathrm{n}} \\
{\left[\frac{\mathrm{m}^{2}}{V \mathrm{~s}}\right]}\end{array}$ & $\begin{array}{c}V_{\mathrm{bi}} \\
{[\mathrm{V}]}\end{array}$ & $\begin{array}{c}R_{\mathrm{S}} \\
{[\Omega]}\end{array}$ & $\begin{array}{c}R_{\mathrm{D}} \\
{[\Omega]}\end{array}$ \\
\hline $\begin{array}{c}\text { GAT1 } \\
\text { 432A/4/LID }\end{array}$ & 4 & 0,3 & 360 & $6,7 \times 10^{16}$ & 3740 & 0,8 & 16 & 16 \\
\hline $\begin{array}{c}\text { THC 302 } \\
\text { No 18 }\end{array}$ & 1 & 0,18 & 800 & $2 \times 10^{17}$ & 2060 & 0,8 & 1,2 & 1,2
\end{tabular}

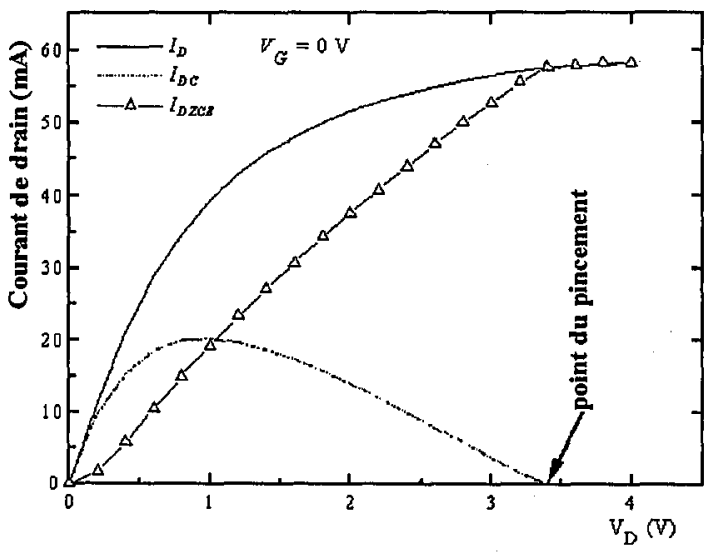

Fig. 4. Variation de différents termes de l'expression (10) pour le transistor GAT1 avec $R_{\mathrm{S}}=R_{\mathrm{D}}=0 \Omega$.

Figure 4 représente la variation des différents termes constituants le courant de drain en fonction de $V_{\mathrm{D}}$ pour le transistor GAT1 $\left(R_{\mathrm{S}}=R_{\mathrm{D}}=0 \Omega\right.$ et $v_{\mathrm{s}}=$ $\left.0,96 \times 10^{7} \mathrm{~cm} / \mathrm{s}\right)$. On constate que $I_{\mathrm{DC}}$ augmente avec $V_{\mathrm{D}}$ jusqu'à ce qu'il atteint une certaine valeur maximale, puis il subit une décroissance jusqu'à ce qu'il s'annule pour une certaine valeur de $V_{\mathrm{D}}$; pour laquelle la ZCE couvre tout le canal $a \leq h$; c'est le régime de pincement. Cette allure traduit bien l'influence des deux 


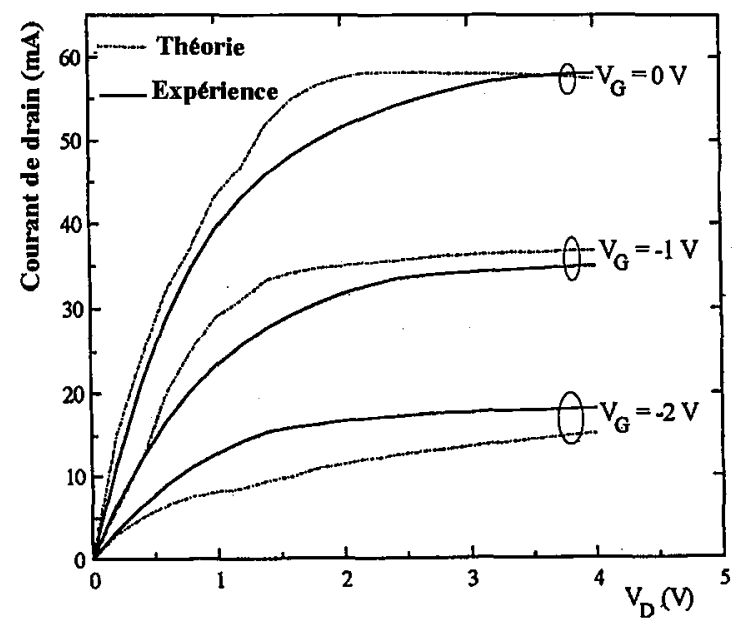

Fig. 5. Comparaison théorie-expérience pour le transistor GAT1 avec $R_{\mathrm{S}}=R_{\mathrm{D}}=0 \Omega$ et $v_{\mathrm{s}}=0,96 \times 10^{7} \mathrm{~cm} \mathrm{~s}^{-1}$.

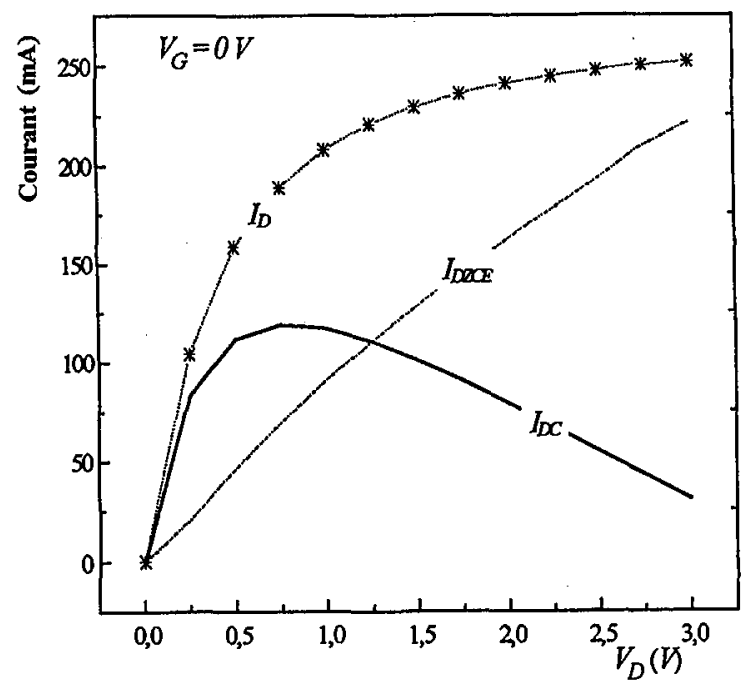

Fig. 6. Variation de différents termes de l'expression (10) pour le transistor THC 302 avec $R_{\mathrm{S}}=R_{\mathrm{D}}=0 \Omega$.

facteurs: la vitesse des porteurs et la zone de déplétion, qui agissent l'un contre l'autre. La deuxième partie du courant de drain $I_{\mathrm{DZCE}}$ augmente également au fur et à mesure que $V_{\mathrm{D}}$ augmente, puis elle se sature en régime de pincement. Les valeurs du courant de drain $I_{\mathrm{D}}=I_{\mathrm{DC}}+I_{\mathrm{DZCE}}$ obtenues sont satisfaisantes, c'est ce que confirme Fig. 5, sur laquelle nous avons représenté une comparaison théorie-expérience pour le même transistor GAT1. Figure 6 montre également la variation des différents termes constituants le courant de drain pour le transistor THC $(L=1 \mu \mathrm{m})$. On peut donner à cette figure la même explication que 


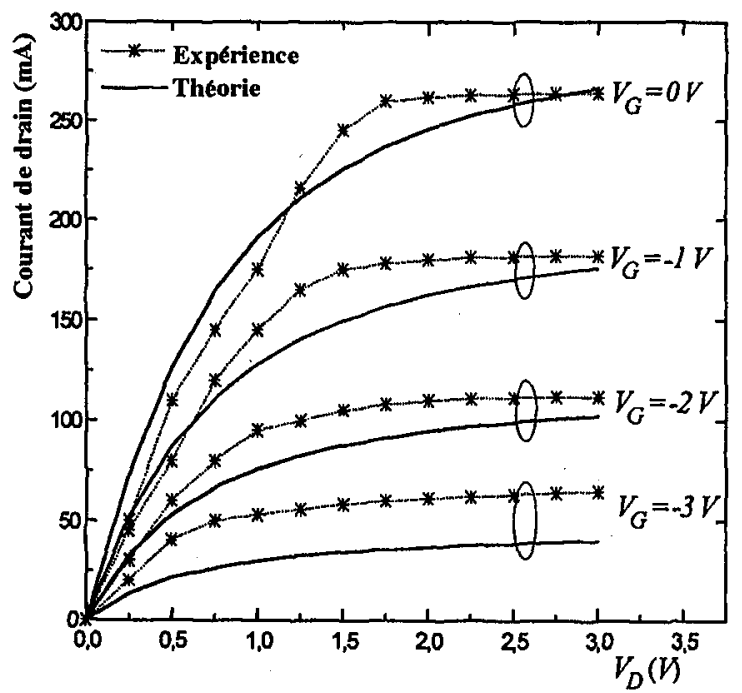

Fig. 7. Comparaison théorie-expérience pour le transistor THC 302 avec $R_{\mathrm{S}}=R_{\mathrm{D}}=$ $1,2 \Omega$ et $v_{\mathrm{s}}=1,42 \times 10^{7} \mathrm{~cm} \mathrm{~s}^{-1}$.

la précédente, et on peut constater en particulier que le courant de drain se sature avant le pincement du canal; ceci est dû au fait que la longueur du canal est courte, ce qui permet au champ électrique d'atteindre des valeurs appréciables rapidement, et par conséquent la saturation rapide de la vitesse des porteurs dans le canal. Ceci veut dire que la saturation du courant de drain dans ce cas est due à la saturation de la vitesse des porteurs. Figure 7 montre une comparaison théorie-expérience pour le même transistor THC, sur laquelle on peut voir le bien fondé de notre approche.

Maintenant on utilise l'expression de la vitesse suivante [2] pour les deux composants à canal court dont les paramètres sont donnés dans Tableau II

$$
\begin{aligned}
& v(E)=\mu_{\mathrm{n}} E-\frac{\left(\mu_{\mathrm{n}} E_{\mathrm{C}}-v_{a}\right) E^{2}}{E_{\mathrm{C}}^{2}} \quad \text { pour } E \leq E_{\mathrm{C}}, \\
& v(E)=v_{a}+\frac{\mu_{b}\left(E-E_{\mathrm{C}}\right)}{1+\mu_{b}\left(E-E_{\mathrm{C}}\right) /\left(v_{\mathrm{sl}}-v_{a}\right)} \quad \text { pour } E \geq E_{\mathrm{C}} .
\end{aligned}
$$

Les résistances parasites sont données dans ce cas [2] par

$$
R_{\mathrm{S}}=\frac{L_{\mathrm{gs}}}{q N_{\mathrm{D}} \mu_{\mathrm{n}} Z a}, \quad R_{\mathrm{D}}=\frac{L_{\mathrm{gd}}}{q N_{\mathrm{D}} \mu_{\mathrm{n}} Z a},
$$

$L_{\mathrm{gs}}$ et $L_{\mathrm{gd}}$ sont les distances grille-source et grille-drain respectivement. Les resultats obtenus pour ces deux transistors sont représentés sur Figs. 8 et 9 , qui représentent une comparaison theorie-expérience. 
TABLEAU II

Paramètres des composants à canal court.

\begin{tabular}{l|c|c}
\hline \hline Paramètres & Composant 1 & Composant 2 \\
\hline$L[\mu \mathrm{m}]$ & 1 & 0,5 \\
$L_{\mathrm{gs}}[\mu \mathrm{m}]$ & 0,79 & 0,47 \\
$L_{\mathrm{gd}}[\mu \mathrm{m}]$ & 0,64 & 0,4 \\
$a[\mu \mathrm{m}]$ & 0,153 & 0,144 \\
$N_{\mathrm{D}}\left[\times 10^{17} \mathrm{~cm}^{-3}\right]$ & 1,17 & 1,31 \\
$\mu_{\mathrm{n}}\left[\mathrm{cm}^{2} \mathrm{~s}^{-1} \mathrm{~V}^{-1}\right]$ & 3435 & 3438 \\
$v_{a}\left[\times 10^{17} \mathrm{~cm} / \mathrm{s}\right]$ & 0,96 & 1,2 \\
$\mu_{b}\left[\mathrm{~cm}^{2} \mathrm{~s}^{-1} \mathrm{~V}^{-1}\right]$ & 2760 & 3000 \\
$v_{\mathrm{sl}}\left[\times 10^{7} \mathrm{~cm} \mathrm{~s}^{-1}\right]$ & 1,69 & 2,5
\end{tabular}

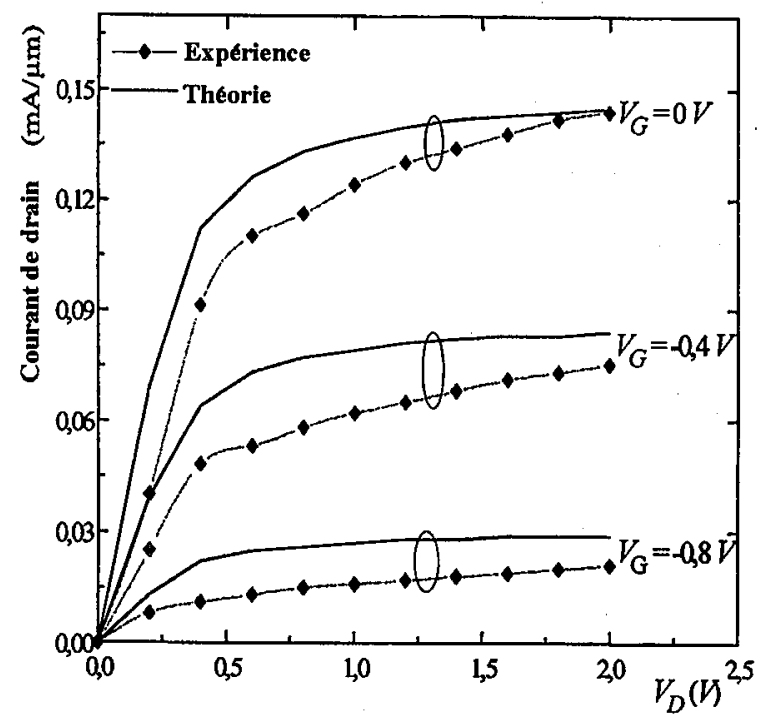

Fig. 8. Comparaison théorie-expérience pour le composant $2(L=0,5 \mu \mathrm{m})$.

L'expression (35) [3] décrit mieux que les deux précédentes la variation de la vitesse des électrons dans le GaAs

$$
v(E)=\frac{\mu_{\mathrm{n}} E+v_{\mathrm{s}}\left(\frac{E}{E_{\mathrm{C}}}\right)^{4}}{1+\left(\frac{E}{E_{\mathrm{C}}}\right)^{4}} .
$$

En fait, l'utilisation de l'expression de $v(E)(35)$ à titre d'exemple pour le transistor GAT1, nous permet d'aboutir à Fig. 10, sur laquelle on peut voir la diminution que subit le courant en régime de saturation. Ceci est dû à la non prise 


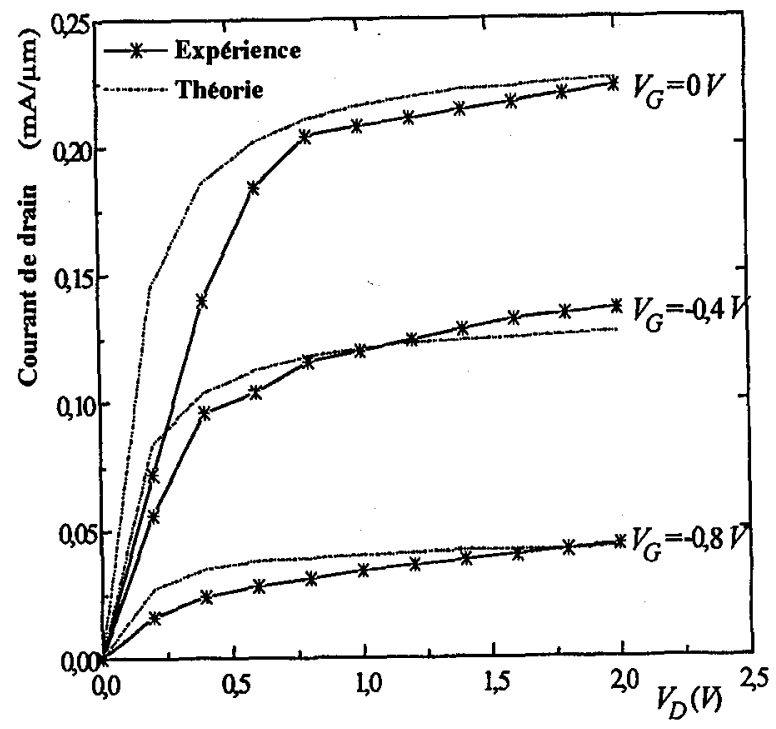

Fig. 9. Comparaison théorie-expérience pour le composant $1(L=1 \mu \mathrm{m})$.

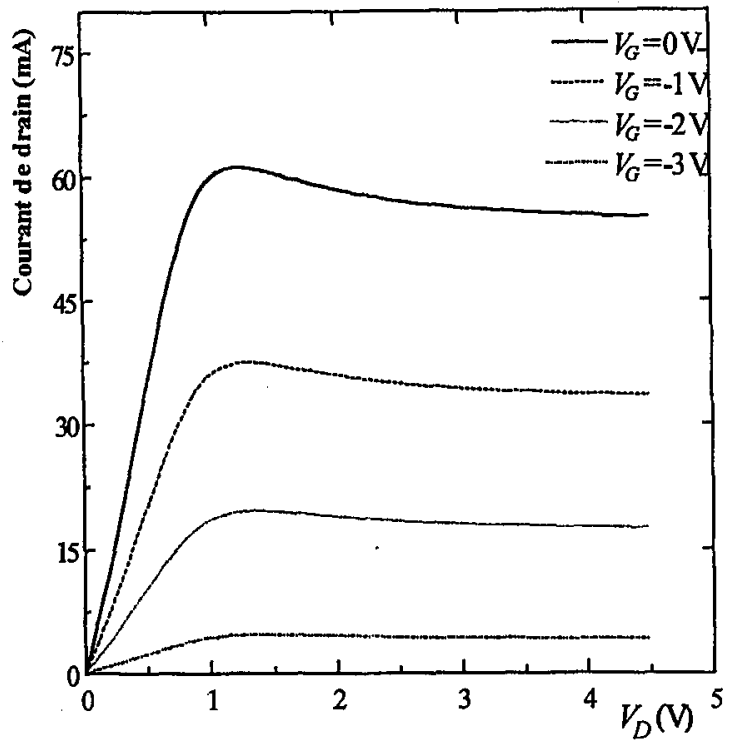

Fig. 10. Variation de $I_{\mathrm{D}}$ avec $V_{\mathrm{D}}$ pour différentes valeurs de $V_{\mathrm{G}}$ pour le transistor GAT1 obtenue en utilisant l'expression de la vitesse (35).

en considération du phénomène d'accumulation des porteurs qui se produit dans le canal.

Figure 11 représente une comparaison théorie-expérience pour le même transistor GAT1, dont les valeurs théoriques sont calculées en tenant compte de (18) 


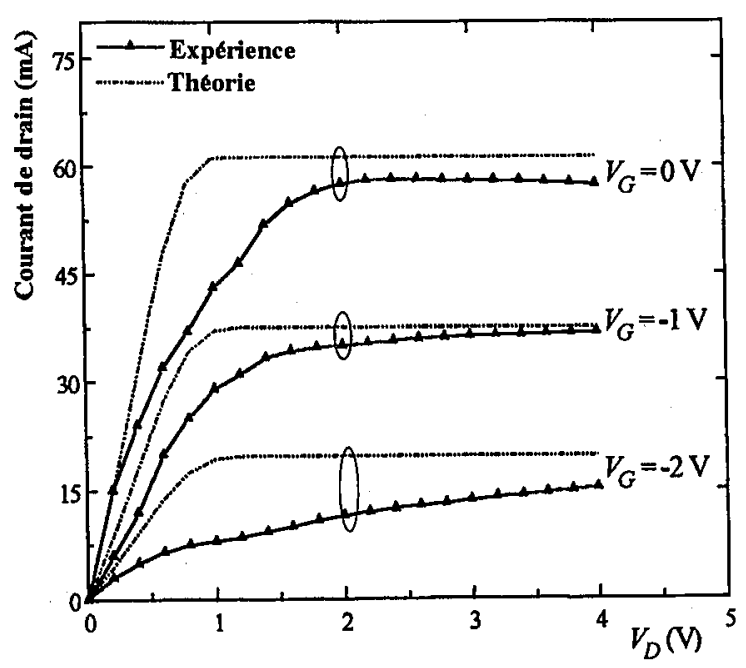

Fig. 11. Comparaison théorie-expérience de $I_{D}\left(V_{D}\right)$ pour le transistor GAT1 obtenue en utilisant l'expression de $v(E)(35)$ et en tenant compte de l'effet d'accumulation des électrons.

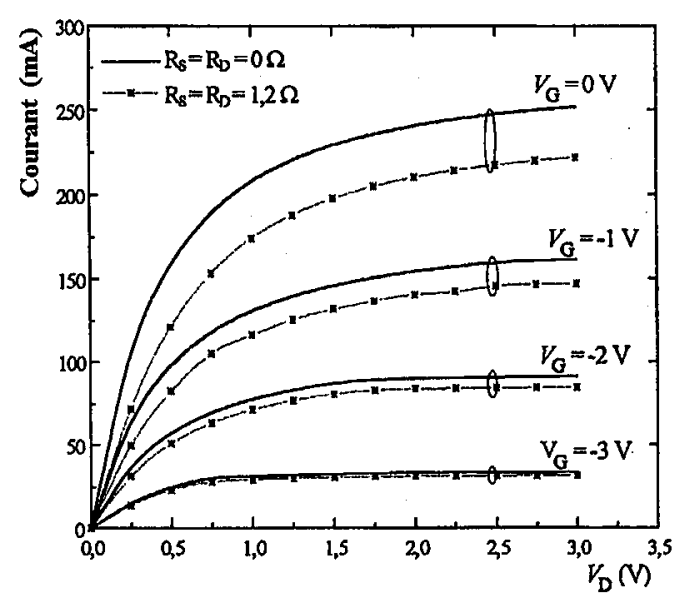

Fig. 12. Comparaison de la variation de $I_{\mathrm{D}}\left(V_{\mathrm{D}}\right)$ dans les deux cas $R_{\mathrm{S}}=R_{\mathrm{D}}=0 \Omega$ et $R_{\mathrm{S}}=R_{\mathrm{D}}=1,2 \Omega$ pour le transistor $\mathrm{THC}$.

et (19). Un accord relativement satisfaisant entre le modèle analytique proposé et les caractéristiques $I-V$ expérimentaux est obtenu. La différence observée entre les valeurs du courants calculées par les différentes expressions de vitesse des électrons, montre la dépendance du modèle avec la loi de la mobilité.

Dans le but de mettre en évidence les effets des résistances parasites $\left(R_{\mathrm{S}}\right.$ et $R_{\mathrm{D}}$ ) sur les caractéristiques $I-V$ du MESFET, les résultats pour le transistor THC 302 , sont présentées sur Fig. 12. On voit clairement que les effets des résistances parasites ne peuvent pas être négligés. Cette figure montre également que l'effet de 


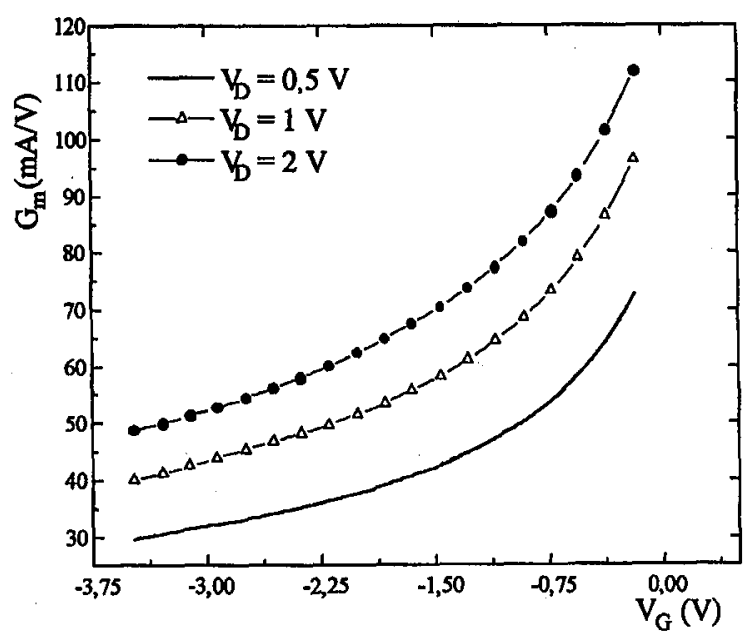

Fig. 13. Variation de la transconductance en fonction de la tension de grille pour le transistor THC.



Fig. 14. Variation de différents termes constituant $C_{\mathrm{G}}$ en fonction de $V_{\mathrm{G}}$ pour le transistor THC.

$R_{\mathrm{S}}$ et $R_{\mathrm{D}}$ est d'autant plus remarquable que $V_{\mathrm{G}}$ est grande, parce que la résistance du canal augmente quand $V_{\mathrm{G}}$ diminue.

Pour le calcul de la fréquence de coupure, on utilise l'expression de $v(E)$ (32) à titre d'exemple pour le transistor THC. Figure 13 illustre la variation de la transconductance $G_{m}\left(V_{\mathrm{G}}\right)$ pour différentes valeurs de $V_{\mathrm{D}}$. La variation de la capacité de grille $C_{G}\left(V_{G}\right)$ est représentée sur Figs. 14 et 15 . Et enfin, Fig. 16 représente la variation de la fréquence de coupure $f_{\mathrm{C}}\left(V_{\mathrm{G}}\right)$ pour le même transistor $\mathrm{THC}$, et pour différentes valeurs de $V_{\mathrm{D}}$. On constate que la fréquence de coupure est 


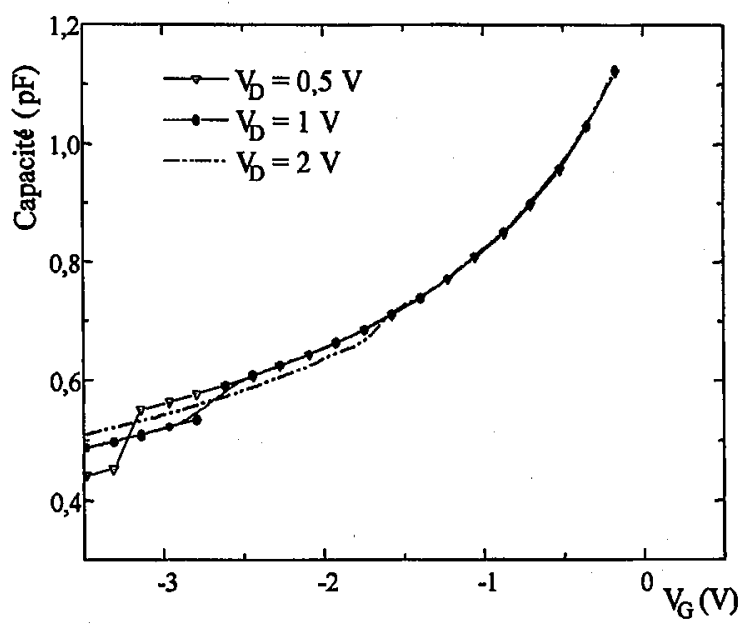

Fig. 15. Variation de $C_{\mathrm{G}}$ en fonction de $V_{\mathrm{G}}$ pour differentes valeurs de $V_{\mathrm{D}}$ pour le transistor THC.

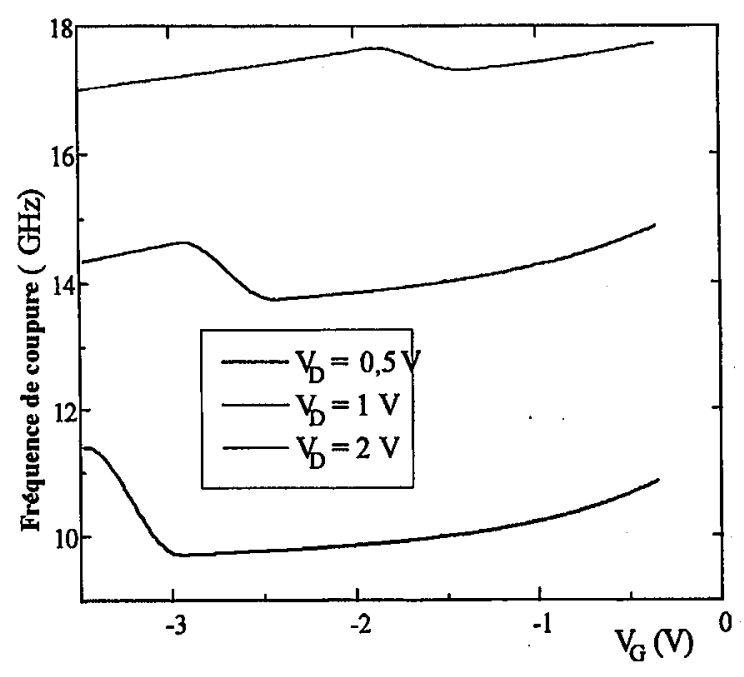

Fig. 16. Variation de $f_{C}\left(V_{G}\right)$ pour le transistor THC.

d'autant plus grande que $V_{\mathrm{D}}$ est grande. On constate aussi que $f_{\mathrm{C}}\left(V_{\mathrm{G}}\right)$ et $C_{\mathrm{G}}\left(V_{\mathrm{G}}\right)$ subissent une variation brusque pour une certaine valeur de $V_{\mathrm{G}}$; ceci provient du passage du fonctionnement du composant du régime de pincement au régime de conduction sans pincement.

\section{Conclusion}

Dans cet article nous avons développé un modèle analytique permettant de décrire le fonctionnement électrique statique du MESFET. Ce modèle permet 
d'obtenir les caractéristiques $I-V$ du MESFET quelque soit sa longueur de canal et quelque soit son régime de fonctionnement (pincement ou non). Nous a vons tenu compte de l'effet de l'injection des électrons dans la ZCE, ce qui nous a permis de calculer la valeur du courant de drain en régime de pincement. La validité de ce modèle dépend essentiellement de l'expression de $v(E)$ que l'on utilise. Dans cet article nous avons calculé également la fréquence de coupure du MESFET en se basant sur ce modèle.

\section{Références}

[1] A. Khiter, diploma thesis, Université de Batna, 1997.

[2] S.P. Chin, Ch.Y. Wu, IEEE Trans. Electron Devices 40, 712 (1993).

[3] S.H. Lo, Ch.P. Le, IEEE Trans. Electron Devices 39, 1564 (1992).

[4] M.A. Khatibzadeh, Ph.D. thesis, North Carolina State University, Raleigh 1987.

[5] M.A. Khatibzadeh, R.J. Trew, IEEE Trans. Microwave 35, 188 (1987).

[6] K. Horio, T. Yamada, IEEE Trans. Electron Devices 46, 648 (1999).

[7] S.M. Sze, Physics of Semiconductor Devices, Wiley, New York 1981.

[8] K. Horio, Y. Fuseya, IEEE Trans. Electron Devices 41, 145 (1994). 